Pacific Journal of Mathematics

CONTINUITY OF SPECTRAL FUNCTIONS AND THE LAKES 


\title{
CONTINUITY OF SPECTRAL FUNCTIONS AND THE LAKES OF WADA
}

\author{
Domingo A. HerRero
}

The functions $\sigma$, mapping a Hilbert space operator $T$ into its spectrum $\sigma(T)$, or $\sigma_{e}$ (defined by $\sigma_{e}(T)=$ essential spectrum of $T$ ), or $\rho_{s-F}^{h}(T)$ mapping $T$ into the set of complex numbers $\lambda$ such that $\lambda-T$ is semi-Fredholm of index $h$, etc, have a very erratic behavior. They are continuous on a dense set of operators and discontinuous on another dense set of operators. It is not completely apparent, however, that all of them are simultaneously continuous on a certain dense subset and simultaneously discontinuous on another dense subset.

In order to prove these two assertions, we shall need some notation. In what follows, $\mathcal{L}(\mathcal{H})$ will denote the algebra of all (bounded linear) operators acting on the complex, separable, infinite dimensional Hilbert space $\mathcal{H}$. By a "spectral function" we shall mean a function mapping an operator $T$ in $\mathcal{L}(\mathcal{H})$ to a certain "natural" subset of its spectrum $\sigma(T)$; more specifically, any of the following functions:

$\sigma$ (spectrum), $\sigma_{l}$ (left spectrum), $\sigma_{r}$ (right spectrum), $\sigma_{l r}$ $\left(\sigma_{l r}(T)=\sigma_{l}(T) \cap \sigma_{r}(T)\right), \sigma_{e}$ (essential spectrum, i.e., the spectrum of $T+\mathscr{H}(\mathcal{H})$ in the quotient Calkin algebra $\mathscr{Q}(\mathcal{H})=\mathcal{L}(\mathcal{H}) / \mathscr{K}(\mathcal{H})$, where $\mathscr{K}(\mathcal{H})$ denotes the ideal of all compact operators), $\sigma_{l e}$ (left essential spectrum), $\sigma_{r e}$ (right essential spectrum), $\sigma_{\text {lre }}$ (Wolf spectrum; $\sigma_{\text {lre }}(T)=$ $\left.\sigma_{l e}(T) \cap \sigma_{r e}(T)\right), \bar{\sigma}_{p}$ (closure of the point spectrum of $T$ ), $\bar{\sigma}_{0}$ (closure of the set of all normal eigenvalues), $\sigma_{B}$ (Browder spectrum; $\sigma_{B}(T)=\sigma(T) \backslash \sigma_{0}(T)$ ), $\sigma_{W}$ (Weyl spectrum; $\left.\sigma_{W}(T)=\bigcap\{\sigma(T+K): K \in \mathscr{K}(\mathcal{H})\}\right), \bar{\rho}_{s-F}^{h}($ defined by $\bar{\rho}_{s-F}^{h}(T)=\{\lambda \in \mathbf{C} ; \lambda-T$ is a semi-Fredholm operator of index $h\}^{-},-\infty \leq y \leq \infty, h \neq 0$ ), or, more generally, $\rho_{s-F}^{\Sigma}$ (defined by $\rho_{s-F}^{\Sigma}(T)=\{\lambda \in \mathbf{C} ; \lambda-T$ is a semi-Fredholm operator and ind $(\lambda-T) \in \Sigma\}^{-}$, for each nonempty subset $\Sigma$ of $\mathbf{Z}^{\prime}=\mathbf{Z}^{*} \backslash\{0\}$, where $\mathbf{Z}^{*}=\mathbf{Z} \cup$ $\{ \pm \infty\})$.

All these functions naturally appear in many problems in Operator Theory. The reader is referred to [3], [4], [5], [6] or [8] for their precise 
definition and to [11] for the definition and properties of the semi-Fredholm operators. It is well-known that

$$
\begin{aligned}
\sigma_{W}(T) & =\sigma(T) \backslash\{\lambda \in \mathbf{C}: \lambda-T \text { is semi-Fredholm of index } 0\} \\
& =\sigma_{e}(T) \cup \rho_{s-F}^{\mathbf{Z}^{\prime}}(T)
\end{aligned}
$$

$[\mathbf{8}],[\mathbf{1 1}]$.

A spectral function $\tau$ maps $\mathcal{L}(\mathcal{H})$ into $\mathcal{C}(\mathbf{C})$, the family of all compact subsets of $\mathbf{C}$, the complex plane. As usual, we make $C(\mathbf{C})$ a complete metric space by defining the (modified) Hausdorff distance $d_{H}$ between two elements of $\mathcal{C}(\mathbf{C})$ by:

(1) If $X, Y \in \mathcal{C}(\mathbf{C}) \backslash\{\varnothing\}$, then

$$
d_{H}(X, Y)=\min \left\{1, \min \left\{\varepsilon>0: X \subset Y_{\varepsilon}, Y \subset X_{\varepsilon}\right\}\right\},
$$

where $X=\{\lambda \in \mathbf{C}$ : $\operatorname{dist}[\lambda, X] \leq \varepsilon\}$, and

(2) $d_{H}(X, \varnothing)=1$ for all nonempty $X$ in $\mathcal{C}(\mathbf{C})$.

The continuity points of the spectral function $\tau$ are then defined in terms of the norm topology of $\mathcal{L}(\mathcal{H})$ and the above mentioned metric structure of $\mathcal{C}(\mathbf{C})$.

In a sequence of remarkable papers, J. B. Conway and B. B. Morrel completely characterized those operators that are points of continuity for each of the functions listed above, except for $\bar{\sigma}_{0}, \sigma_{B}$ and $\rho_{s-F}^{\Sigma}$ (see [3], [4], [5]). On the other hand, it is not difficult to check, by using the results of these papers, that $\sigma_{B}$ is continuous at $T$ if and only if $\sigma_{W}$ is continuous at $T$ and $\sigma_{B}(T)=\sigma_{W}(T)$. Furthermore, by using the same kinds of arguments, it is possible to prove the following.

THEOREM 1. (i) $\bar{\sigma}_{0}$ is continuous at $A \in \mathcal{L}(\mathcal{H})$ if and only if $\sigma(A)=$ $\left[\rho_{s-F}^{\mathbf{Z}^{\prime}}(A) \cup \sigma_{0}(A)\right]^{-}$and $\bar{\sigma}_{0}(A)=\partial \sigma(A)$.

(ii) If $\Sigma$ is a nonempty subset of $\mathbf{Z}^{\prime}$, then $\rho_{s-F}^{\Sigma}$ is continuous at $A \in \mathcal{L}(\mathcal{H})$ if and only if $\sigma_{\text {lre }}(A) \subset \rho_{s-F}^{\Sigma}(A)$.

In each case the sufficiency of the given condition follows from standard arguments based only on the upper semicontinuity of separate parts of the spectrum [8, Chapter 1], [11, Chapter IV] and the stability properties of the semi-Fredholm operators (same references), so that these conditions are actually sufficient in any Banach space (not necessarily a Hilbert space!). The necessity is much more difficult to check and depends on results of approximation of operators developed strictly for the Hilbert space case, as the Apostol-Morrel simple models [2] (see also [1]). 
It was observed in [3] that both, $\sigma$ and $\sigma_{W}$, are continuous on a dense subset and discontinuous on a dense subset (this last result depending on the proof of Theorem 4 in [7]).

This note is devoted to the proof of the following (much stronger) results:

THEOREM 2. $\mathcal{L}(\mathcal{H})$ contains a dense subset $\Gamma_{c}$ such that all the spectral functions mentioned above are continuous at each point of $\Gamma_{c}$.

THEOREM 3. $\mathcal{L}(\mathcal{H})$ contains a dense subset $\Gamma_{d}$ such that all the spectral functions mentioned above are discontinuous at each point $\Gamma_{d}$.

1. The lakes of Wada. The name of the title is a classical construction of Point Set Topology, producing an indecomposable continuum. More precisely, the construction produces three nonempty disjoint open subsets, $\Omega_{0}, \Omega_{1}$ and $\Omega_{2}$, of $C$ with the property that the three of them have the same boundary, and this common boundary $X\left(=\partial \Omega_{0}=\partial \Omega_{1}=\partial \Omega_{2}\right)$ is a compact set. The details of the construction can be found, for example, in [12] [10, pp. 143-145]. The case of denumerably many open sets (instead of just three) follows by exactly the same argument and yields the following result:

LEMMA 4. Let $\Delta$ be a nonempty compact connected subset of $\mathbf{C}$ such that $\Delta=(\text { interior } \Delta)^{-}$. Then there exists a denumerble family $\left\{\Omega_{h}\right\}_{h \in \mathbf{Z}^{*}}$ of pairwise disjoint simply connected open subsets of $\Delta$ such that $\cup_{h \in \mathbf{Z}^{*}} \Omega_{h}$ is dense in $\Delta$ and $\partial \Omega_{h}=\partial \Omega_{0}$ for all $h$.

CoROllaRY 5. Let $\Delta$ and $\left\{\Omega_{h}\right\}_{h \in \mathbf{Z}^{*}}$ be as in Lemma 4. There exists $L_{\Delta}$ in $\mathcal{L}(\mathcal{H C})$ such that $\sigma\left(L_{\Delta}\right)$ is the disjoint union of $\Delta \backslash \Omega_{0}$ and a sequence $\left\{\lambda_{j}\right\}_{j=1}^{\infty}$ contained in $\Omega_{0}$ such that

$$
\left\{\lambda_{j}\right\}^{-}=\left\{\lambda_{j}\right\} \cup \partial \Omega_{0}, \quad \sigma_{e}\left(L_{\Delta}\right)=\left(\Omega_{\infty} \cup \Omega_{-\infty}\right)^{-},
$$

each $\lambda_{j}$ is a normal eigenvalue of $L_{\Delta}$ of multiplicity 1 and for each $h \in \mathbf{Z}^{\prime}$ and each $\lambda$ in $\Omega_{h}, \lambda-L_{\Delta}$ is a semi-Fredholm operator such that $\operatorname{ind}\left(\Lambda-L_{\Delta}\right)=h$ and $\min \left\{\operatorname{dim} \operatorname{ker}\left(\lambda-L_{\Delta}\right), \operatorname{dim} \operatorname{ker}\left(\lambda-L_{\Delta}\right)^{*}\right\}=0$.

Proof. Decompose $\mathcal{H}=\bigoplus_{h \in \mathbf{Z}^{*}} \mathcal{H}_{h}$ (orthogonal direct sum), where $\mathcal{H}_{h}$ is an infinite dimensional subspace. Let $\left\{\lambda_{j}\right\}_{j=1}^{\infty}$ be a denumerable subset of $\Omega_{0}$ such that dist $\left[\lambda_{j}, \partial \Omega_{0}\right] \rightarrow 0(j \rightarrow \infty)$ and $\left\{\lambda_{j}\right\}^{-}=\left\{\lambda_{j}\right\} \cup \partial \Omega_{0}$ and define $N_{0} \in \mathcal{L}\left(\mathcal{H}_{0}\right)$ by the equations $N_{0} e_{j}=\lambda_{j} e_{j}(j \geq 1)$ with respect to some orthonormal basis $\left\{e_{j}\right\}_{j=1}^{\infty}$ of $\mathcal{H}_{0}$. 
If $\Omega$ is a nonempty bounded open set such that $\Omega=\operatorname{interior}\left(\Omega^{-}\right)$, then we define $M(\Omega)=$ "multiplication by $\lambda$ " on the space $L^{2}(\Omega, d x d y)$ and $M_{+}(\Omega)=M(\Omega) \mid B^{2}(\Omega)$, where $B^{2}(\Omega)$ is the $L^{2}$-closure of the rational functions in $\lambda$ with poles outside $\Omega^{-}$. For each $h<0$, we define $N_{h}=M_{+}\left(\Omega_{h}\right)^{(h \mid)}$ (= direct sum of $|h|$ copies of $M_{+}\left(\Omega_{h}\right)$ ), acting in the usual fashion on the orthogonal direct sum $B^{2}\left(\Omega_{h}\right)^{(|h|)}$ of $|h|$ copies of the Hilbert space $B^{2}\left(\Omega_{h}\right)$. Similarly, for each $j>0$, we define $N_{h}=$ $\left[M_{+}\left(\Omega_{h}^{*}\right)^{*}\right]^{(h)}$, where $\Omega^{*}=\{\bar{\lambda}: \lambda \in \Omega\}$.

Clearly, $B^{2}\left(\Omega_{h}\right)^{(h)}$ is isometrically isomorphic to $\mathcal{H}_{h}$ for each $h<0$, and $B^{2}\left(\Omega_{h}^{*}\right)^{(h)}$ is isometrically isomorphic to $\mathcal{H}_{h}$ for each $h>0$. Thus, we can directly assume that $N_{h} \in \mathcal{L}\left(\mathcal{H}_{h}\right)$ for all $h \in \mathbf{Z}^{*}$. Now it is easy to check (by using, for instance, the results of [8, Chapter IV]) that equation

$$
L=\bigoplus_{h \in \mathbf{Z}^{*}} N_{h}
$$

actually defines an operator acting on $\mathcal{H}$ with the desired properties.

The semi-Fredholm domain of $T \in \mathcal{L}(\mathcal{H C})$ is the open set $\rho_{s-F}(T)=$ $\{\lambda \in \mathbf{C}: \lambda-T$ is semi-Fredholm $\}\left(=\mathbf{C} \backslash \sigma_{l r e}(T)\right)$. Assume that

$$
\sigma(T)=\left(\Gamma_{1} \cup \Gamma_{2} \cup \cdots \cup \Gamma_{r}\right) \cup\left\{\mu_{1}, \mu_{2}, \ldots, \mu_{n}\right\} \cup\left\{\nu_{1}, \nu_{2}, \ldots, \nu_{p}\right\}
$$

(disjoint union), where

(1) $\Gamma_{t}$ is the closure of the bounded open set interior $\Gamma_{t} \subset \rho_{s-F}(T)$; $\operatorname{ind}(\lambda-T) \neq 0$ and $\min \left\{\operatorname{dim} \operatorname{ker}(\lambda-T), \operatorname{dim} \operatorname{ker}(\lambda-T)^{*}\right\}=0$ for all $\lambda \in$ interior $\Gamma_{t}(t=1,2, \ldots, r ; r<\infty)$;

(2) $\partial\left(\cup_{t=1}^{r} \Gamma_{t}\right)$ is the union of finitely many pairwise disjoint smooth Jordan curves $\gamma_{1}, \gamma_{2}, \ldots, \gamma_{m}$;

(3) $\left\{\mu_{1}, \mu_{2}, \ldots, \mu_{n}\right\}$ is a finite set of isolated points of $\sigma_{e}(T)$; and

(4) $\left\{\nu_{1}, \nu_{2}, \ldots, \nu_{p}\right\}=\sigma_{0}(T)$.

Let $\eta>0$ be small enough to guarantee that $\sigma(T)_{\eta}$ has exactly the same number of components as $\sigma(T)$ and let $\Delta_{j}=\left(\gamma_{j}\right)_{\eta} \backslash($ interior $\sigma(T))$ for $j=1,2, \ldots, m$, and $\Delta_{j}=\left\{\lambda:\left|\lambda-\mu_{j-m}\right|<\eta\right\}$ for $j=m+1, m+$ $2, \ldots, m+n$. For each $j, j=1,2, \ldots, m+n$, we choose an operator $L_{\Delta}$, defined exactly as in Corollary 5 (with $\Delta$ replaced by $\Delta_{j}, j=1,2, \ldots, m+$ $n$ ) and define $L_{\eta}=\bigoplus_{j=1}^{m+n} L_{\Delta_{j}}$.

Combining the "easy part" of the results of [3], [4], [5] with Theorem 1 , we conclude that

Proposition 6. Let $T$ and $L_{\eta}$ be as above; then all the spectral functions considered here are continuous at $T \oplus L_{\eta}$. 
2. Simultaneous continuity. Let $A \in \mathcal{L}(\mathcal{H})$ and let $\varepsilon>0$ be given. The main result of [2] says that there exists an operator $A_{\varepsilon} \in \mathcal{E}(\mathcal{F C})$ such that $\left\|A-A_{\varepsilon}\right\|<\varepsilon$ and

$$
A_{\varepsilon} \simeq\left(\begin{array}{lll}
S_{+} & * & * \\
0 & M & * \\
0 & 0 & S_{-}
\end{array}\right),
$$

where $\simeq$ denotes unitary equivalence, $\sigma\left(S_{+}\right), \sigma(M)$ and $\sigma\left(S_{-}\right)$are pairwise disjoint, $A_{\varepsilon}$ is similar to $S_{+} \oplus M \oplus S_{-}, M$ is a normal operator with finite spectrum, $\sigma\left(S_{+}\right)=\left\{\lambda \in \rho_{s-F}\left(S_{+}\right) \text {: } \operatorname{ind}\left(\lambda-S_{+}\right)<0\right\}^{-}$, $\rho_{s-F}\left(S_{+}\right) \cap \sigma\left(S_{+}\right)=$interior $\sigma\left(S_{+}\right)$and $\operatorname{dim} \operatorname{ker}\left(\lambda-S_{+}\right)=0$ for all $\lambda \in \rho_{s-F}\left(S_{+}\right), \sigma\left(S_{-}\right)=\left\{\lambda \in \rho_{s-F}\left(S_{-}\right): \operatorname{ind}\left(\lambda-S_{-}\right)>0\right\}^{-}, \rho_{s-F}\left(S_{-}\right)$ $\cap \sigma\left(S_{-}\right)=$interior $\sigma\left(S_{-}\right)$and $\operatorname{dim} \operatorname{ker}\left(\lambda-S_{-}\right)=0$ for all $\lambda \in$ $\rho_{s-F}\left(S_{-}\right)$, and $\partial\left[\sigma\left(S_{+}\right) \cup \sigma\left(S_{-}\right)\right]$consists of finitely many pairwise disjoint smooth Jordan curves.

Thus, $S_{+} \oplus M \oplus S_{-}$has exactly the same form as the operator $T$ of Proposition 6. For each $\eta>0$ small enough, we define $\left(S_{+} \oplus M \oplus S_{-}\right) \oplus$ $L_{\eta}$ as in Proposition 6. It readily follows that $S_{+} \oplus M \oplus S_{-} \oplus L_{\eta}$ is a point of continuity for all spectral functions,. A fortiori, so is every operator similar to $S_{+} \oplus M \oplus S_{-} \oplus L_{\eta}$.

Following [1], let us write $R \rightarrow B$ to indicate that the operator $B$ is the norm limit of operators similar to $R$. According to the same reference, there is a normal operator $M$ such that $\sigma\left(M_{\eta}\right)=\sigma\left(L_{\eta}\right)$ and $L_{\eta} \rightarrow M_{\eta}$. It follows that $S_{+} \oplus M \oplus S_{-} \oplus L_{\eta} \rightarrow S_{+} \oplus M \oplus S_{-} \oplus M_{\eta}$; moreover, $S_{+} \oplus M \oplus S_{-}$can be uniformly approximated by operators similar to $S_{+} \oplus M \oplus S_{-} \oplus M_{\eta}$. (Consider a sequence of such operators corresponding to a decreasing sequence $\left\{\eta_{n}\right\}_{n=1}^{\infty}$ such that $\eta_{n}>0$, as $n \rightarrow \infty$.)

Hence, $S_{+} \oplus M \oplus S_{-}$is the limit of a sequence of points of continuity. Therefore, so is the operator $A_{\varepsilon}$ (similar to $S_{+} \oplus M \oplus S_{-}$).

Since $\varepsilon$ can be chosen arbitrarily small we conclude that $A$ can be uniformly approximated by a sequence $\left\{A_{n}\right\}_{n=1}^{\infty}$ such that all the spectral functions considered in the Introduction are simultaneously continuous at $A_{n}$, for each $n \geq 1$.

The proof of Theorem 2 is now complete.

REMARKS. (i) The spectral radius and the essential spectral radius are also continuous at each point of the set $\Gamma_{c}$ described by Theorem 2 (see [3]).

(ii) It is tempting to think that any "natural" spectral function is necessarily continuous at the points of $\Gamma_{c}$. However, this is not true at all. 
Namely, the spectral function defined by

$$
\overline{\sigma_{e}^{0}}(T)=\left\{\{\lambda\}:\{\lambda\} \text { is a component of } \sigma_{e}(T)\right\}^{-}
$$

(that is, the closure of the union of those components of $\sigma_{e}(T)$ which consist of a single point), which plays a very important role in the work of Conway and Morrel, is continuous nowhere! (This can be deduced, for example, from the main result of [1].)

\section{Simultaneous discontinuity.}

LEMMA 7. Let $R$ be a normal operator such that $\sigma(R)=\{\lambda:|\lambda| \leq 1\}$ and $\sigma_{p}(R)=\varnothing$. All the spectral functions considered in the Introduction are discontinuous at $R$.

Proof. Let $Q$ be a quasinilpotent operator such that $Q^{k}$ is compact for no value of $k \geq 1$, and let $N_{0}$ be defined as in the proof of Corollary 5 with $\lambda_{j}=2^{-j}, j=1,2 \ldots$ According to [1], $Q \oplus N_{0} \rightarrow R$, whence it readily follows that all " $\sigma$-functions" (that is, $\sigma, \sigma_{B}, \sigma_{l}, \sigma_{e}$, etc.) are discontinuous at $R$ because every neighborhood of $R$ contains an operator similar to $Q \oplus N_{0}$.

Similarly, if $S$ is a semi-Fredholm operator such that $\sigma(S)=\sigma(R)$ and ind $S=h \in \mathbf{Z}^{\prime}$, then $S \rightarrow R$, whence we conclude that $\rho_{s-F}^{\Sigma}$ is discontinuous at $R$ for all possible sets $\Sigma$.

According to [7] (Theorem 4 and its proof), given $A$ in $\mathcal{L}(\mathcal{H})$, a point $\lambda \in \sigma_{e}(A) \cap \partial \sigma(A), \varepsilon>0$ and $R \in \mathcal{L}(\mathcal{H}),\|R\| \leq 1$, there exists $A(\lambda, \varepsilon, R)$ unitarily equivalent to

$$
\left(\begin{array}{cc}
\mu+\delta R & * \\
0 & A^{\prime}
\end{array}\right)
$$

such that $\sigma_{e}\left(A^{\prime}\right)=\sigma_{e}(A), 0<\operatorname{dist}\left[\mu, \sigma\left(A^{\prime}\right)\right]<\varepsilon,|\lambda-\mu|<\varepsilon, 0<\delta<\varepsilon$ and $\|A-A(\lambda, \varepsilon, R)\|<\varepsilon$; furthermore, $A(\lambda, \varepsilon, R)$ is similar to $(\mu+\delta R)$ $\oplus A^{\prime}$, so that if the spectral function $\tau$ is discontinuous at $R$, then $\tau$ is also discontinuous at $A(\lambda, \varepsilon, R)$.

By Lemma 7, $R$ can be chosen so that all the spectral functions are discontinuous. The proof of Theorem 3 is now complete.

REMARKS. (i) If $\lambda$ is chosen so that $|\lambda|=\max \left\{|\xi|: \xi \in \sigma_{e}(A)\right\}$ (= the essential spectral radius of $A$ ), then we obtain a bonus result: The set $\Gamma_{d}$ of Theorem 3 can be chosen so that the essential spectral radius is also discontinuous at each point of $\Gamma_{d}$. 
Can we choose $\Gamma_{d}$ so that the spectral radius, $\operatorname{sp}(A)=\max \{|\lambda|$ : $\lambda \in \sigma(A)$, is also discontinuous at the points of $\Gamma_{d}$ ? Definitely: NO. Indeed, if $\mu \in \sigma_{0}(A),|\mu|=\operatorname{sp}(A)$ and $\varepsilon>0$, then the upper semicontinuity of separate parts of the spectrum and the continuity properties of the Riesz-Dunford functional calculus (see, for example, [8, Corollary 1.6]) imply that $\sigma_{0}(B) \cap\{\lambda \in \mathbf{C}:|\lambda-\mu|<\varepsilon\} \neq \varnothing$ and therefore $\operatorname{sp}(B)>$ $\operatorname{sp}(A)-\varepsilon$ for all $B$ close enough to $A$. Since the spectral radius is an upper semicontinuous function of its argument, we conclude that $\mathrm{sp}$ is continuous at $A$.

Combining this observation with the main result of [9], we obtain the following

Proposition 8. For each complex Banach space $\mathfrak{X}, \mathcal{L}(\mathfrak{X})$ contains an open dense subset $\Phi$ such that sp is continuous at every point of $\Phi$.

\section{REFERENCES}

[1] C. Apostol, D. A. Herrero and D. Voiculescu, The closure of the similarity orbit of a Hilbert space operator, Bull. Amer. Math. Soc. (New Series), 6 (1982), 421-426.

[2] C. Apostol and B. B. Morrel, On uniform approximation of operators by simple models, Indiana Univ. Math. J., 16 (1977), 427-442.

[3] J. B. Conway and B. B. Morrel, Operators that are points of spectral continuity, Integral Equations and Operator Theory, 2 (1979), 174-198.

[4] Operators that are points of spectral continuity. II, Integral Equations and Operator Theory, 4 (1981), 459-503.

[5] Operators that are points of spectral continuity. III, Integral Equations and Operator Theory, 6 (1983), 319-344.

[6] P. R. Halmos, A Hilbert Space Problem Book, D. Van Nostrand, Princeton. New Jersey, 1967.

[7] D. A. Herrero, On multicyclic operators, Integral Equations and Operator Theory, 1 (1978), 57-102.

[8] Approximation of Hilbert Space Operators, Pitman Publ. Inc., London-Boston-Toronto, 1982.

[9] D. A. Herrero and N. Salinas, Operators with disconnected spectra are dense, Bull. Amer. Math. Soc., 78 (1972), 525-526.

[10] J. G. Hocking and G. S. Yound, Topology, Addison Wesley Publ. Co., London, 1961.

[11] T. Kato, Perturbation Theory for Linear Operators, Springer-Verlag, New York, 1966.

[12] K. Yoneyama, Theory of continuous sets of points I, Tohoku Math. J., 12 (1917), 43-153.

Received August 31, 1982 and in revised form January 5, 1983. This research has been partially supported by a Grant of the National Science Foundation. 



\section{PACIFIC JOURNAL OF MATHEMATICS \\ EDITORS}

Donald BaBBITT (Managing Editor)

University of California

Los Angeles, CA 90024

Hugo RossI

University of Utah

Salt Lake City, UT 84112

C. C. Moore and Arthur Ogus

University of California

Berkeley, CA 94720
J. DugundiI

Department of Mathematics

University of Southern California

Los Angeles, CA 90089-1113

R. FINN and H. SAMELSON

Stanford University

Stanford, CA 94305

\section{ASSOCIATE EDITORS}
R. ARENS
E. F. BECKENBACH
B. H. NeUMANN
F. WOLF
K. YoshidA (1906-1982)

\section{SUPPORTING INSTITUTIONS}

\author{
UNIVERSITY OF ARIZONA \\ UNIVERSITY OF BRITISH COLUMBIA \\ CALIFORNIA INSTITUTE OF TECHNOLOGY \\ UNIVERSITY OF CALIFORNIA \\ MONTANA STATE UNIVERSITY \\ UNIVERSITY OF NEVADA, RENO \\ NEW MEXICO STATE UNIVERSITY \\ OREGON STATE UNIVERSITY
}

\author{
UNIVERSITY OF OREGON \\ UNIVERSITY OF SOUTHERN CALIFORNIA \\ STANFORD UNIVERSITY \\ UNIVERSITY OF HAWAII \\ UNIVERSITY OF TOKYO \\ UNIVERSITY OF UTAH \\ WASHINGTON STATE UNIVERSITY \\ UNIVERSITY OF WASHINGTON
}

The Supporting Institutions listed above contribute to the cost of publication of this Journal, but they are not owners or publishers and have no responsibility for its content or policies.

Mathematical papers intended for publication in the Pacific Journal of Mathematics should be in typed form or offset-reproduced (not dittoed), double spaced with large margins. Please do not use built up fractions in the text of the manuscript. However, you may use them in the displayed equations. Underline Greek letters in red, German in green, and script in blue. The first paragraph must be capable of being used separately as a synopsis of the entire paper. In particular it should contain no bibliographic references. Please propose a heading for the odd numbered pages of less than 35 characters. Manuscripts, in triplicate, may be sent to any one of the editors. Please classify according to the scheme of Math. Reviews, Index to Vol. 39. Supply name and address of author to whom proofs should be sent. All other communications should be addressed to the managing editor, or Elaine Barth, University of California, Los Angeles, California 90024.

There are page-charges associated with articles appearing in the Pacific Journal of Mathematics. These charges are expected to be paid by the author's University, Government Agency or Company. If the author or authors do not have access to such Institutional support these charges are waived. Single authors will receive 50 free reprints; joint authors will receive a total of 100 free reprints. Additional copies may be obtained at cost in multiples of 50 .

The Pacific Journal of Mathematics is issued monthly as of January 1966. Regular subscription rate: $\$ 132.00$ a year (6 Vol., 12 issues). Special rate: $\$ 66.00$ a year to individual members of supporting institutions.

Subscriptions, orders for numbers issued in the last three calendar years, and changes of address should be sent to Pacific Journal of Mathematics, P.O. Box 969, Carmel Valley, CA 93924, U.S.A. Old back numbers obtainable from Kraus Periodicals Co., Route 100, Millwood, NY 10546.

The Pacific Journal of Mathematics ISSN 0030-8730 is published monthly by the Pacific Journal of Mathematics at P.O. Box 969, Carmel Valley, CA 93924. Application to mail at Second-class postage rates is pending at Carmel Valley, California, and additional mailing offices. Postmaster: Send address changes to Pacific Journal of Mathematics, P. O. Box 969, Carmel Valley, CA 93924.

PUBLISHED BY PACIFIC JOURNAL OF MATHEMATICS, A NON-PROFIT CORPORATION

Copyright $\odot 1984$ by Pacific Journal of Mathematics 


\section{Pacific Journal of Mathematics}

\section{Vol. 113, No. $2 \quad$ April, 1984}

Alan Adolphson, On the Dwork trace formula ...................257

Amos Altshuler and Leon Steinberg, Enumeration of the quasisimplicial

3 -spheres and 4-polytopes with eight vertices .................. 269

Kenneth R. Goodearl, Cancellation of low-rank vector bundles .......... 289

Gary Fred Gruenhage, Ernest A. Michael and Yoshio Tanaka, Spaces

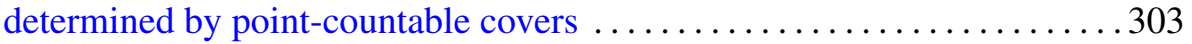

Charles Lemuel Hagopian, Atriodic homogeneous continua .......... 333

David Harbater, Ordinary and supersingular covers in characteristic $p$. . . 349

Domingo Antonio Herrero, Continuity of spectral functions and the lakes

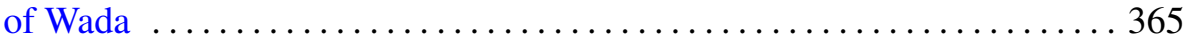

Donald William Kahn, Differentiable approximations to homotopy resolutions and framed cobordism ....................... 373

K. McGovern, On the lifting theory of finite groups of Lie type $\ldots \ldots \ldots . \ldots 383$

C. David (Carl) Minda, The modulus of a doubly connected region and the geodesic curvature-area method ............................. 395

Takuo Miwa, Complexes are spaces with a $\sigma$-almost locally finite base $\ldots . .407$

Ho Kuen Ng, Finitely presented dimension of commutative rings and modules

Roger David Nussbaum, A folk theorem in the spectral theory of

$C_{0}$-semigroups

J. S. Okon, Prime divisors, analytic spread and filtrations

Harold Raymond Parks, Regularity of solutions to elliptic isoperimetric problems

R. Sitaramachandra Rao and M. V. Subba Rao, Transformation formulae for multiple series

Daniel Ruberman, Imbedding punctured lens spaces and connected sums

Uri Srebro, Deficiencies of immersions 